\title{
Paper 100
}

\section{Optimum Design of Cable-Stayed Bridges with Imprecise Data}

\author{
L.M.C. Simões and J.H. Negrão \\ Department of Civil Engineering, \\ University of Coimbra-Polo II, Coimbra, Portugal
}

\begin{abstract}
This work presents the Two-phase method for fuzzy optimization of cable stayed bridges. The minimization problem is stated as the minimization of stresses, displacements and bridge cost. A finite-element approach is used for structural analysis. It includes a direct analytic sensitivity analysis module, which provides the structural behaviour responses to changes in the design variables. An equivalent multicriteria approach is used to solve the non-differentiable, non-linear optimization problem, turning the original problem into the sequential minimization of unconstrained convex scalar functions, from which a Pareto optimum is obtained.In the first phase the fuzzy solution is obtained by using the Level Cuts Method and in the second phase the crisp solution, which maximizes the membership function of fuzzy decision-making, is found by using the Bound Search Method. Illustrative numerical examples are solved.
\end{abstract}

Keywords: Fuzzy optimization, cable-stayed bridges, structures.

\section{Introduction}

Cable-stayed bridges are large and expensive structures that are now being widely used. The trend to increase the spans and the advent of innovative erection techniques make thus desirable the development of computational tools to assist on the preliminary design stage and/or erection control, which today relies mostly on the design staff expertise. The authors have been involved in the last few years in such a project, which led to the development of a programme dealing with the aspects of design which can be objectively expressed by a numerical merit measure. The optimization can be stated as that of the minimization of structural cost or volume, and the maximum stresses throughout the structure. Additional objectives are aimed at the deflections or displacements and to guarantee that the design variables are at least specified minimum values. This work started with the shape 
and sizing optimization by using a $2 \mathrm{D}$ finite-element model for the analysis. The problem was extended to three-dimensional analysis and the consideration of erection stages under static loading [1] Seismic effects were considered in the optimization both by a modal-spectral approach and a time-history based procedure [2]. In most of the previous studies, a grid solution was adopted for modelling the deck, with stiffening girders supporting transverse beams, although box-girder sections were employed [3]. Prestressing design variables were also considered for the problems of optimal correction of cable forces during erection [4].

In design and optimization problems material constants, loading and structure geometry are usually considered as given data, but in real world assumed values do not correspond with actual ones. Therefore there may be differences between nominal and real geometry, materials may behave in a different way than the assumed one, and constant loading may actually vary during the structure lifetime. All of this is accounted by safety factors, which amplify load magnitude, or reduce material strength, leading in general to over-conservative structures.

As an alternative to safety factors one may try to describe the uncertain data and use this information during the optimization, which in general leads to better results in term of optimal design. Probabilistic description is nowadays common and very simple up to very sophisticated PDF can be used to describe uncertain parameters. However these procedures face difficulties when being implemented in engineering applications. This lead to non-probabilistic description of uncertainty, in particular the fuzzy-set based analysis and the worst condition produced on the constraints by a certain load condition also termed anti-optimization [5]. The Two-Phase Method for fuzzy optimization of structures is based on the fuzzy-set method is proposed in this work. In the first phase, the sequential fuzzy solution is obtained by using the Level Cuts Method, in which a fuzzy optimization problem is transformed into a series of ordinary optimization problems using different $\alpha$-level cuts in fuzzy constraints so as to determine a fuzzy optimization domain in the design space. This procedure has been suggested in [6]. In the second phase, the particular crisp solution is obtained by the Bound Search Method, in which having obtained the supremum and the infimum of the sequential fuzzy solution the particular optimum level $\alpha^{*}$ is found using the bound search so as to provide a crisp optimization solution in the design space.

\section{Fuzziness in Structural Design}

A fuzzy information problem of structures can be stated as: Find the design vector $\underline{x}$ which minimizes the objective function $\mathrm{W}(\underline{\mathrm{x}})$ subject to fuzzy constraints on performance characteristics and dimensions. This is a fuzzy mathematical programming problem, which can

be expressed in a standard form as follows (MP1):

$$
\begin{array}{lll}
\min W(\underline{x}) & & \\
s t & g_{j}(\underline{x}) \mathbb{b _ { j }}{ }^{U} & j=1,2, \ldots, m-1 \\
& g_{j}(\underline{x}) D b_{j}{ }^{L} & j=m, \ldots, p
\end{array}
$$


where the " " and " " symbols indicate that the constraints contain fuzzy information, and $b_{j}{ }^{\mathrm{U}}, b_{j}{ }^{\bar{L}}$ are allowable upper and lower limits of the jth constraint respectively.

The membership function $\mu_{\mathrm{j}}(\underline{\mathrm{x}})$ of the fuzzy allowable interval may be characterized as shown in Figure 1 . where $b_{j}{ }^{L}$ and $b_{j}^{U}$ are respectively the lower and upper limits of the allowable interval for the highest (most rigorous) design level.

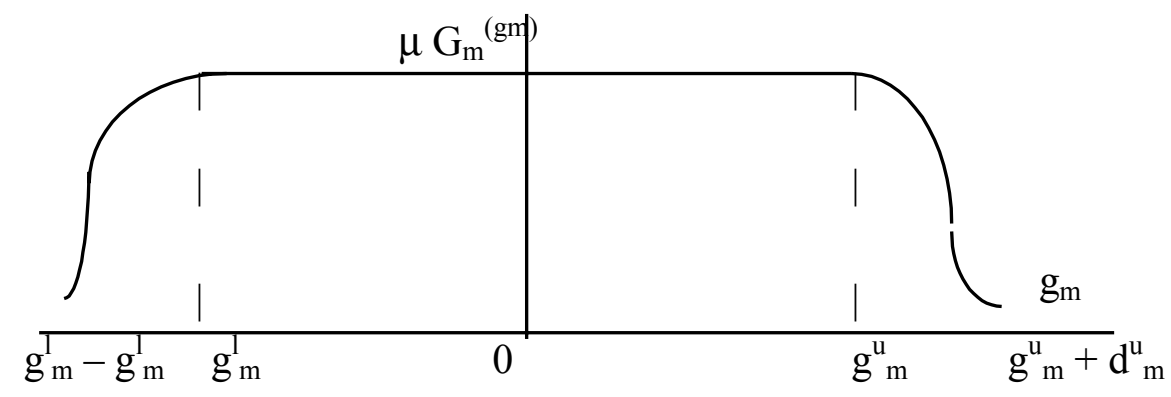

Figure 1

These may even be more strict than the specifications Codes (to be chosen by engineering requirements). $d_{j}{ }^{L}$ and $d_{j}^{U}$ are lengths of transition stages, namely the permissible deviations or tolerances for the lower and upper limits. Thus, MP1 can be rewritten in the following form (MP2):

$$
\begin{array}{lll}
\min W(\underline{x}) & \\
\text { st } & g_{j}(\underline{x}) \leq b_{j}{ }^{U}+d_{j}^{U} & j=1,2, \ldots, m-1 \\
& g_{j}(\underline{\mathbf{x}}) \geq b_{j}{ }^{L}-d_{j}^{L} & j=m, \ldots, p
\end{array}
$$

A proper function may be selected for the transition stage curves of the membership function in the light of the character of the physical variable $g_{j}$. Usually, inclined straight lines may be adopted for simplification.

Membership degree $\mu_{\mathrm{j}}(\underline{\mathrm{x}})$ may be defined as "degree of satisfaction" of the fuzzy constraint. When $\mu_{\mathrm{j}}(\underline{\mathrm{x}})=1$, the constraint is satisfied completely: when $\mu_{\mathrm{j}}(\underline{\mathrm{x}})=0$ is not: while its value lies between 0 and 1, the constraint is satisfied to the relevant degree.

An alternative formulation consists of expressing the objective function and constraints as normalized goals. If some reference cost $\mathrm{W}_{\mathrm{o}}$ is specified, the goals may be written in the form

$$
\begin{array}{ll}
\mathrm{g}_{\mathrm{o}}(\underline{\mathbf{x}})=\mathrm{W}(\underline{\mathbf{x}}) / \mathrm{W}_{\mathrm{o}}-1 \leq 0 & \\
\mathrm{~g}_{\mathrm{j}}(\underline{\mathbf{x}}) /\left(\mathrm{b}_{\mathrm{j}}^{\mathrm{U}}+\mathrm{d}_{\mathrm{j}}^{\mathrm{U}}\right)-1 \leq 0 & \mathrm{j}=1,2, \ldots, \mathrm{m}-1 \\
1-\mathrm{g}_{\mathrm{j}}(\underline{\mathbf{x}}) /\left(\mathrm{b}_{\mathrm{j}}^{\mathrm{L}}-\mathrm{d}_{\mathrm{j}}^{\mathrm{L}}\right) \leq 0 & \mathrm{j}=\mathrm{m}, \ldots, \mathrm{p}
\end{array}
$$

The objective is to minimize all of theses goals over variables $\underline{\mathbf{x}}$. This can be achieved by the minimax optimization (MP3):

$$
\operatorname{Min}_{\underline{\mathbf{x}}} \operatorname{Max}_{\mathrm{i}}<\mathrm{g}_{\mathrm{o}}(\underline{\mathbf{x}}), \ldots, \mathrm{g}_{\mathrm{j}}(\underline{\mathbf{x}}), \ldots, \mathrm{g}_{\mathrm{p}}(\underline{\mathbf{x}})>
$$




\section{Two-Phase Method}

\subsection{First phase (Level Cuts Method)}

For the sake of simplicity only the method of solving problems with fuzzy constraints is discussed in this work. If the membership function of inclined straight lines may be adopted, as shown in Figure 2, MP2 can be transformed into a nonfuzzy mathematical programming at $\alpha$-level as follows (MP4):
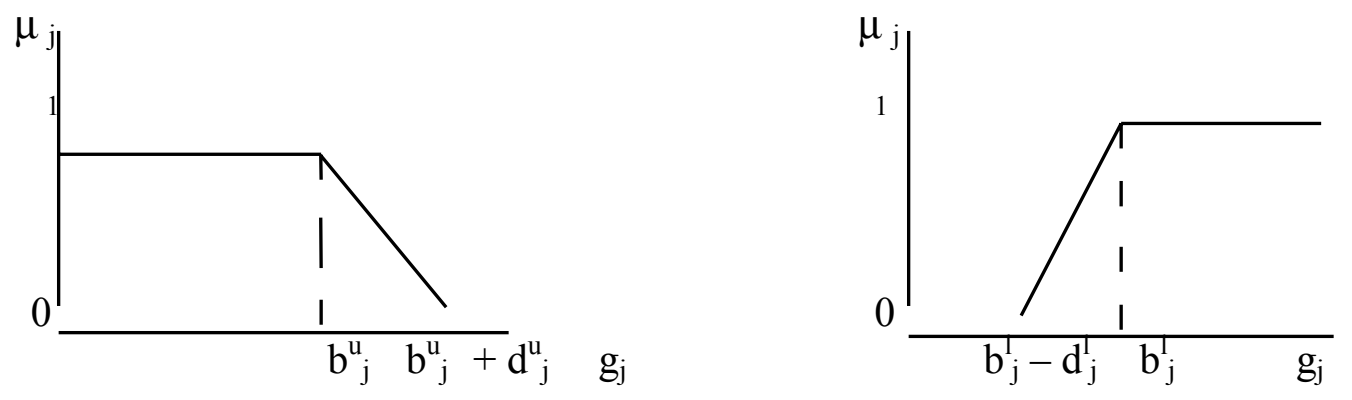

Figure 2: Membership function

$$
\begin{aligned}
& \min W(\underline{x}) \\
& \text { st } g_{j}(\underline{x}) \leq b_{j}^{U}+d_{j}^{U}(1-\alpha) \quad j=1,2, \ldots, m-1 \\
& g_{j}(\underline{x}) \geq b_{j}^{L}-d_{j}^{L} \quad(1-\alpha) \quad j=m, \ldots, p \\
& \alpha \varepsilon[0,1]
\end{aligned}
$$

This is a parametric mathematical programming in $\alpha \in[0,1]$, which can be solved by means of an algorithm for optimization so as to determine the fuzzy optimization solution $\underline{\mathbf{x}}^{*}(\alpha)$ and $\mathrm{W}\left(\underline{\mathbf{x}}^{*}(\alpha)\right)$ with different $\alpha$ values. It is noted that a fuzzy optimization problem may have mixed fuzzy and crisp constraints. In this case we shall accept tolerances only on the realization of fuzzy constraints, but crisp ones will completely satisfied. As shown in Figure 3, the $\mathrm{W}\left(\underline{\mathbf{x}}^{*}(\alpha)\right)$ curve defines a fuzzy solution to the fuzzy optimization problem of structures, which is a monotone increasing function of $\alpha$.

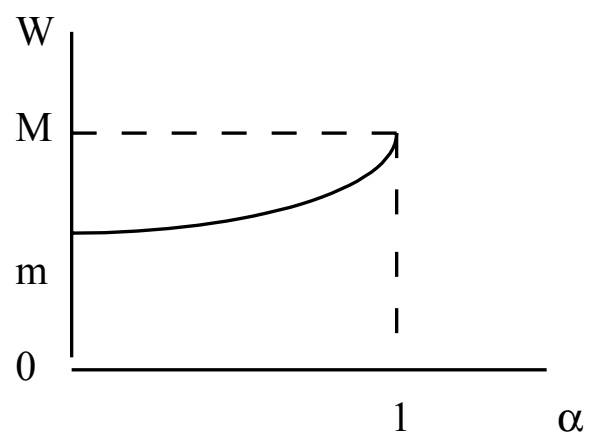

Figure 3: Fuzzy solution 


\subsection{Second phase (Bound Search Method)}

The Bellman-Zadeh [7] criterion of decision-making in a fuzzy environment gives the grade of membership of a decision specified by variables $\mathrm{x}$ as

$$
\mu_{\mathrm{d}}(\underline{\mathbf{x}})=\operatorname{Min}_{\mathrm{i}} \mu_{\mathrm{i}}(\underline{\mathbf{x}})
$$

where $i$ ranges over the complete set of constraints. The fuzzy constraints $\mathrm{C}$ and the fuzzy goal G in MP4 are defined as fuzzy sets in the space of alternatives, characterized by their membership functions $\mu_{C}$ and $\mu_{D}$ respectively. Generally speaking, the fuzzy decision $\mathrm{D}$ characterized by its membership function $\mu_{\mathrm{D}}$ may be viewed as the intersection of the fuzzy constraints and the fuzzy goal.

The optimal decision is to select the best alternative from those contained in the fuzzy decision space, which maximizes the membership function of the fuzzy decision, i.e.:

$$
\mu_{\mathrm{D}}\left(\underline{\mathbf{x}}^{*}\right)=\max \underset{\mathrm{x} \in \mathrm{R}}{ } \mathrm{n} \mu_{\mathrm{D}}(\underline{\mathbf{x}})
$$

In order to illustrate the above principle, consider one fuzzy goal $\mathrm{G}$ with one fuzzy constraint $\mathrm{C}$. The membership functions $\mu_{\mathrm{G}}, \mu_{\mathrm{C}}$ and their intersection $\mu_{\mathrm{D}}$ are plotted for this case in Figure 4. This figure also shows that the point A represents the optimal decision which has the maximum degree of membership in the fuzzy decision set.

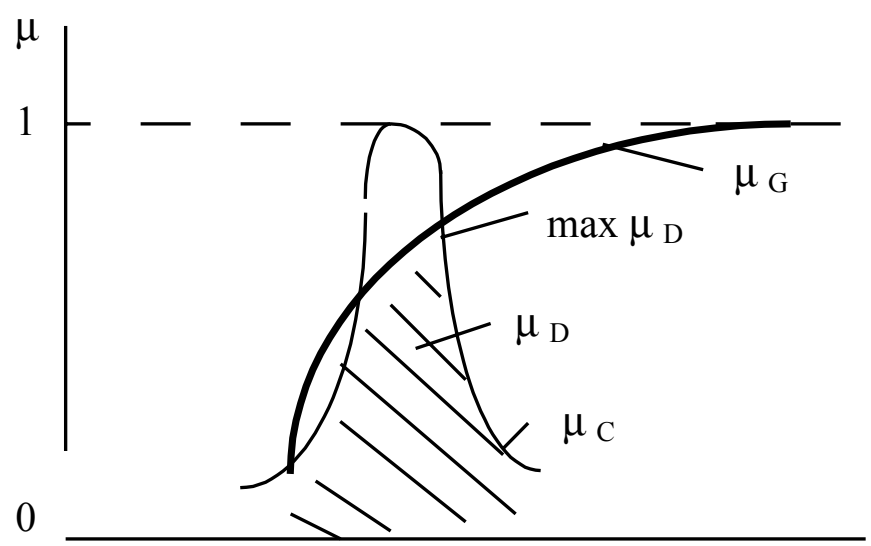

Figure 4

From eq.(7), the particular optimum level $\alpha^{*}$ and the optimum point $\underline{\mathbf{x}}^{*}$ are such that:

$$
\mu_{\mathrm{G}}\left(\underline{\mathbf{x}}^{*}\right)=\max _{\mathrm{x} \in \mathrm{C} \alpha} \mu_{\mathrm{G}}(\underline{\mathbf{x}})
$$

where ${ }_{C \alpha}$ is the $\alpha^{*}$-level cut of the fuzzy constraint set $\mathrm{C}$. 
Now it is necessary to establish the fuzzy goal $\mu_{\mathrm{G}}(\underline{\mathbf{x}})$ and its upper and lower limits. It can be seen from Figure 3 that the supremum and the infimum in the sequential fuzzy solution are given by

$$
\begin{aligned}
& \mathrm{M}=\mathrm{W}\left(\underline{\mathbf{x}}^{*}(1)\right)=\min _{\mathrm{x} \in \mathrm{C} 1} \mathrm{~W}(\underline{\mathbf{x}}) \\
& \mathrm{m}=\mathrm{W}\left(\underline{\mathbf{x}}^{*}(0)\right)=\min _{\mathrm{x} \in \mathrm{C} 0} \mathrm{~W}(\underline{\mathbf{x}})
\end{aligned}
$$

where $\mathrm{C}_{1}, \mathrm{C}_{0}$ are the level cuts of $\alpha=1$ and 0 of the fuzzy constraint set $\mathrm{C}$.

In the problem of finding $\underline{\mathbf{x}}$ which maximizes the objective function $\mathrm{W}(\underline{\mathbf{x}})$ subject to fuzzy constraints, the fuzzy goal is: $\mu_{\mathrm{G}}(\underline{\mathbf{x}})=\mathrm{W}(\underline{\mathbf{x}}) / \mathrm{M}$. Similarly, in a fuzzy optimization problem of structures to find $\mathrm{x}$ which minimizes the objective function $\mathrm{W}(\underline{\mathbf{x}})$ subject to fuzzy constraints, the fuzzy goal can be established as follows:

$$
\mu_{\mathrm{G}}(\underline{\mathbf{x}})=\mathrm{m} / \mathrm{W}(\underline{\mathbf{x}})
$$

As expected, this fuzzy goal shows the full membership $\left(\mu_{\mathrm{G}}=1\right)$ is obtained when $\mathrm{W}$ reaches its infimum $\mathrm{m}$; as $\mathrm{W}$ increases $\mu_{\mathrm{G}}$ approaches the non-membership $\left(\mu_{\mathrm{G}}=0\right)$. Clearly, the upper and lower limits of the fuzzy goal are given by:

$$
\begin{aligned}
& \mu_{\mathrm{G}}^{\mathrm{U}}=1 \\
& \mu_{\mathrm{G}}{ }^{\mathrm{L}}=\mathrm{m} / \mathrm{M}
\end{aligned}
$$

The optimum level can be derived from equation (8) as:

$$
\alpha^{*}=\mu_{\mathrm{G}}\left(\underline{\mathbf{x}}^{*}\left(\alpha^{*}\right)\right)
$$

\subsection{Multiple $\alpha$}

This procedure can be extended to problems exhibiting multiple $\alpha$, each associated with a different type of imprecision. The optimum level for each $\alpha_{i}$ is found by conducting bound searches. With this optimum set of $\alpha$, the fuzzy optimum solution is then obtained.

\section{Structural Relations}

\subsection{Finite element analysis}

The finite element based open code MODULEF was used as the basic tool for structural analysis, because code availability was a fundamental requirement for further developments. Out of the several element types included in the element library of the programme, only the FE required for two- and three-dimensional models of cable-stayed bridges were retained and adapted to specific needs. These were 2D and 3D bar and beam (Euler-Bernoulli formulation) elements and 4- and 8noded serendipity plate-membrane (Reissner-Mindlin formulation) elements. 


\subsection{Design variables}

The structural response of a cable-stayed bridge is conditioned by a large number of parameters, concerning cross-sectional shapes and dimensions, overall bridge geometry, applied prestressing forces, deck-to-pylon connections, etc. Some of them play only a limited role on the bridge behaviour while others, such as the cable pattern and prestressing forces, are of major importance for both the safety and serviceability purposes. Three types of design variables were considered: sizing, shape and mechanical. Sizing design variables are cross-sectional characteristics of bar, beam and plate elements, such as web height, flange width, plate thickness, etc. Changes of such variables do not imply the need for remeshing. Shape design variables produce geometry changes that require nodal co-ordinates update or even complete remeshing. Other design variables can be characterized as hybrid, because they define both the box-girder cross-section shape and the deck geometry, requiring co-ordinates updating only. Finally, the fixed-end prestressing force is a mechanical design variable not related to any geometric quantity. The currently available types are shown in Figure 5.

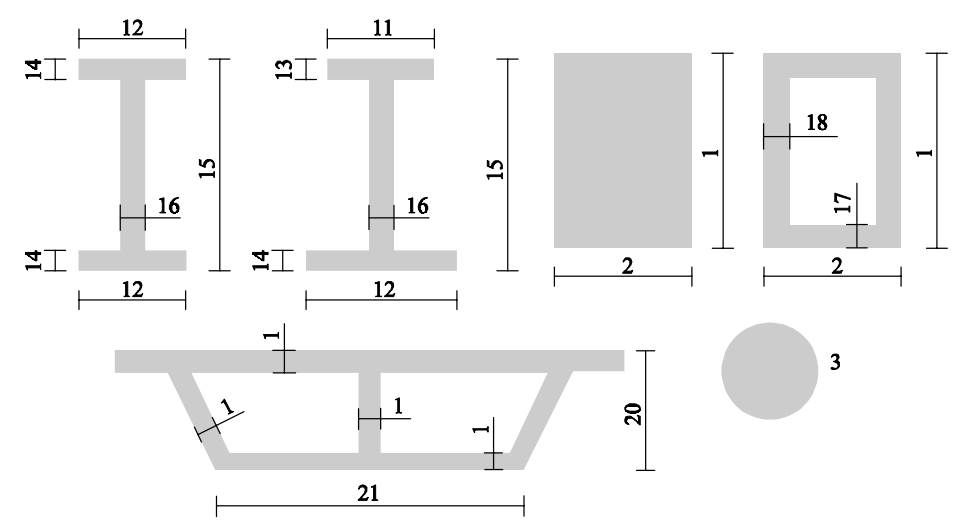

Figure 5: Sizing design variables

All these types play complementary roles in the process of design optimization. Sizing design variables directly provide for cost/volume decrease. Shape and mechanical design variables have a neglectable direct relation to structural cost but allow for better stress distributions, which in turn lead to further decreases in sizing variables. Prestressing force design variables are essential for achieving acceptable solutions when deflections are considered in the dead load condition.

\subsection{Sensitivity analysis}

The analytic direct method was adopted for the purpose of sensitivity analysis, given the availability of the code, the discrete structural pattern and the large number of constraints under control. For ordinary linear statics problems, derivatives of kinematic constraints (displacements) are provided by solving a pseudo-load system. 


$$
\underline{K} \frac{\partial \underline{u}}{\partial \mathrm{xi}}=\frac{\partial \underline{P}}{\partial \mathrm{xi}}-\frac{\partial \underline{K}}{\partial \mathrm{xi}} \underline{u}=\underline{Q}_{\mathrm{vi}_{\mathrm{i}}}
$$

where $\underline{\mathbf{Q}}_{i}$ is the virtual pseudo-load vector of the system with respect to the $i$ th design variable.

The stress derivatives are accurately determined from the chain derivation of the finite element stress matrix

$$
\begin{aligned}
& \underline{\sigma}=\underline{D} \underline{B} e \underline{u} e \\
& \frac{\partial \underline{\sigma}}{\partial \mathrm{x}_{i}}=\frac{\partial\left(\underline{D} \underline{B}{ }_{\mathrm{e}}\right)}{\partial \mathrm{xi}_{\mathrm{i}}} \underline{u_{\mathrm{e}}}+\underline{D} \underline{B} \underline{\underline{\mathrm{e}}} \frac{\partial \underline{u_{\mathrm{e}}}}{\partial \mathrm{x}_{\mathrm{i}}}
\end{aligned}
$$

\subsection{Optimization}

Pareto's economic principle is gaining increasing acceptance to multi-objective optimization problems. In minimization problems a solution vector is said to be Pareto optimal if no other feasible vector exists that could decrease one objective function without increasing at least another one. The optimum vector usually exists in practical problems and is not unique.

Cross-sectional (and geometric) design variables are considered, represented by $\mathrm{x}_{\mathrm{i}}$ and $\mathrm{z}_{\mathrm{i}}$, respectively, and the global design variable vector is

$$
\underline{\mathbf{x}}=\left\{\mathrm{x}_{1}, \mathrm{x}_{2}, \mathrm{x}_{3}, \ldots, \mathrm{x}_{\mathrm{n}}, \mathrm{z}_{\mathrm{i}+1}, \mathrm{Z}_{\mathrm{i}+2}, \ldots, \mathrm{Z}_{\mathrm{N}}\right\}
$$

Bounds must be set for these variables in order to achieve executable solutions and required aesthetic characteristics.The overall objective of cable-stayed bridges design is to achieve an economic and yet safe solution. In this study it is not intended to include all factors influencing the design economics. One of the factors conventionally adopted is the cost of material used. A second set of goals arises from the requirement that the stresses should be as small as possible.

The optimization method described in the next section requires that all these goals should be cast in a normalized form.If some reference $\operatorname{cost} \mathrm{V}_{\mathrm{o}}$ is specified, this goal can be written in the form

$$
\mathrm{g}_{1}(\mathrm{x}, \mathrm{y})=\mathrm{V}(\mathrm{x}, \mathrm{y}) / \mathrm{V}_{\mathrm{o}}-1 \leq 0
$$

Another set of goals arises from the imposition of lower and upper limits on the sizing variables, namely minimum cable cross sections to prevent topology changes and exequible dimensions for the stiffness girder and pylons cross sections:

$$
\begin{aligned}
& \mathrm{g}_{2}\left(\mathrm{x}_{\mathrm{i}}\right)=-\mathrm{x}_{\mathrm{i}} / \mathrm{x}_{\mathrm{L}}+1 \leq 0 \\
& \mathrm{~g}_{3}\left(\mathrm{x}_{\mathrm{i}}\right)=\mathrm{x}_{\mathrm{i}} / \mathrm{x}_{\mathrm{U}}-1 \leq 0
\end{aligned}
$$

where $x_{i}$ is the $i$-th sizing variable and $x_{L}$ and $x_{U}$ its lower and upper bounds. Similar bounds must be considered for the geometric design variables:

$$
\begin{aligned}
& \mathrm{g}_{4}\left(\mathrm{Z}_{\mathrm{k}}\right)=-\mathrm{Z}_{\mathrm{k}} / \mathrm{Z}_{\mathrm{L}}+1 \leq 0 \\
& \mathrm{~g}_{\mathrm{s}}\left(\mathrm{Z}_{\mathrm{k}}\right)=\mathrm{Z}_{\mathrm{k}} / \mathrm{Z}_{\mathrm{U}}-1 \leq 0
\end{aligned}
$$


where $\mathrm{z}_{\mathrm{k}}$ is the $\mathrm{k}$-th geometric variable and $\mathrm{z}_{\mathrm{U}}, \mathrm{z}_{\mathrm{L}}$ are its upper and lower bounds, respectively. Additional bounds are set when geometric design variables are considered, to ensure that no geometry violation occurs when these design variables are updated. For example, when design variables of types 4 or 5 (Figure 6) are considered, their total length cannot exceed the side span length. If $i$ and $j$ are the positions of those design variables in $\underline{\mathbf{X}}$ vector, we write the condition

$$
\left(\mathrm{Z}_{\mathrm{i}}+\mathrm{z}_{\mathrm{j}}\right) / \mathrm{L}_{\mathrm{s}}-1 \leq 0
$$

where $L_{s}$ stands for side span length.
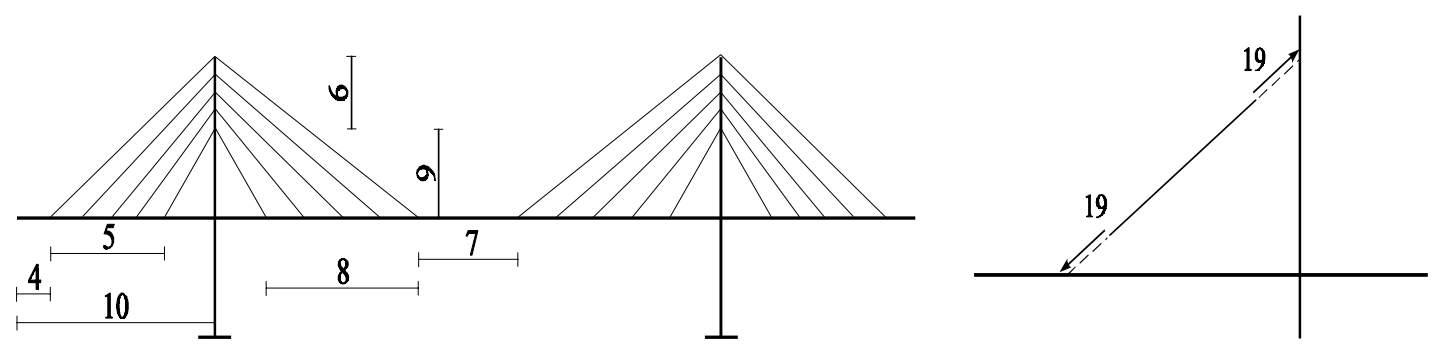

Figure 6: Shape design variables

Additional goals may be established in order to ensure the desired geometric requirements during the optimization process (mesh discretization, ratios of variation of cable spacing on deck and pylons, etc). For these the chosen approach was to initially supply all the necessary information, by means of a geometry coefficients set describing such conditions.

The objective is to minimize all of these objectives over sizing and geometry variables $\underline{\mathbf{X}}$. This problem is discontinuous and non-differentiable and is therefore hard to solve. However, by using an entropy-based approach, Templeman has shown that its solution is equivalent to that of an unconstrained convex scalar function, depending only on one control parameter, which may be solved by conventional quasi-Newton methods. This parameter must be steadily increased through the optimization process. The scalar function is very similar to that of KreisselmeyerStainhauser, derived for control problems:

$$
\mathrm{F}(\underline{x})=\frac{1}{\rho} \cdot \ln \left[\sum_{\mathrm{j}=1}^{\mathrm{M}} \mathrm{e}^{\rho(g(\underline{x}))}\right]
$$

Problem (18) is unconstrained and differentiable which, in theory, gives a wide choice of possible numerical solution methods. However, since the goal functions $\mathrm{g}_{\mathrm{j}}(\mathrm{x}, \mathrm{z})$ do not have explicit algebraic form in most cases, the strategy adopted was to solve (18) by means of an iterative sequence of explicit approximation models. An explicit approximation can be formulated by taking Taylor series expansions of all the goal functions $g_{j}(x, z)$ truncated after the linear term. This gives: 


$$
\operatorname{Min} \quad \mathrm{F}(\underline{x})=\frac{1}{\rho} \cdot \ln \left[\sum_{\mathrm{j}=1}^{\mathrm{M}} \mathrm{e}^{\rho\left(\mathrm{g}_{0}(\underline{\underline{x}})+\sum_{i=1}^{N} \frac{\partial \mathrm{goj}(\underline{x})}{\partial \mathrm{x}_{\mathrm{i}}} \mathrm{dx}\right)}\right]
$$

where $\mathrm{N}$ and $\mathrm{M}$ are respectively the number of sizing plus geometric design variables and the number of goal functions. $g_{0 j}$ and $\partial g_{o j} / \partial x_{i}$ are the goals and their derivatives evaluated for the current design variable vector $\left(\mathrm{x}_{0}, \mathrm{z}_{\mathrm{o}}\right)$, at which the Taylor series expansion is made.

Solving (19) for particular numerical values of $g_{o j}$ forms only one iteration of the complete solution of problem (18). The solution vector $\left(\mathrm{x}_{1}, \mathrm{z}_{1}\right)$ of such an iteration represents a new design which must be analysed and gives new values for $\mathrm{g}_{1 \mathrm{j}}$, $\partial \mathrm{g}_{1 j} / \partial \mathrm{x}_{\mathrm{i}}$ and $\left(\mathrm{x}_{1}, \mathrm{z}_{1}\right)$, to replace those corresponding to $\left(\mathrm{x}_{0}, \mathrm{z}_{\mathrm{o}}\right)$ in (19). Iterations continue until changes in the design variables become small. During these iterations the control parameter $\rho$ must not be decreased to ensure that a multiobjective solution is found.

\section{Numerical Example}

A numerical example with sizing design variables only and without erection stages will be presented next. The model represented in Figure 7 was considered. It consists of a symmetric three-span cable-stayed bridge. Monosymmetric I-shaped cross-sections are prescribed for the stiffening girders, while the pylons are made up of steel plates defining a rectangular hollow cross section. I-shaped transverse beams support the wearing surface. Three load cases, corresponding to live load on either side, central or whole span, were considered.

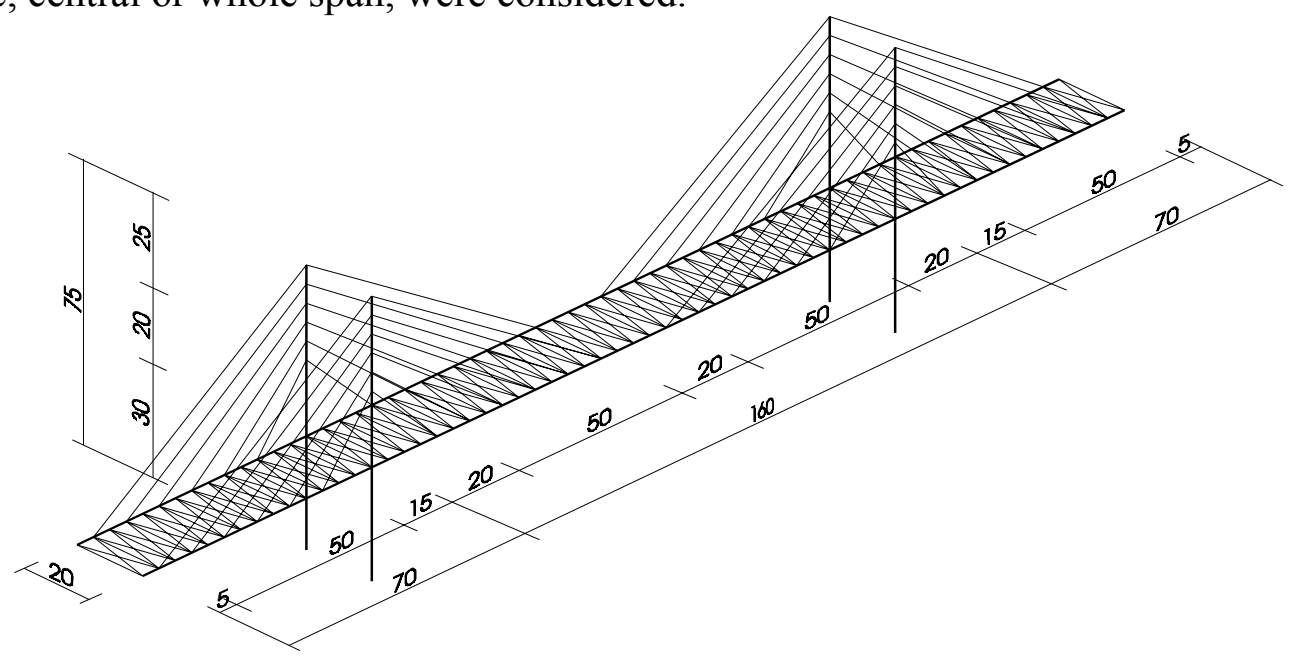

Figure 7: Geometry of the bridge model

Fuzzy goals were generated involving Young's modulus, stress limits and loading. The influence of geometric design variables in the optimum cost is quite limited. Moreover both geometric and sizing design variables in steel cable stayed bridges are strictly controlled and their values are assumed to be deterministic. 
Allowable stresses of $180 \mathrm{MPa}$ were assumed for the deck and pylon elements and $475 \mathrm{MPa}$ for the stays.

Tolerance of $40 \mathrm{MPa}$ for the deck and pylon elements and $50 \mathrm{MPa}$ for the stays were employed to fuzzify the stress limits. A membership function of inclined straight lines is adopted and (18) will become a parametric program. In the first phase, by solving (18) with different $\alpha$ values yield deterministic designs together with a level of acceptability with respect to the stress limits fuzziness. In the second phase, the crisp solution is obtained by the iterative bound search method described in 3.2 .

$$
\mathrm{M}=\mathrm{W}(\alpha=1)=159.52 \quad \mathrm{~m}=\mathrm{W}(\alpha=0)=140.88 \quad 0.883 \leq \alpha \leq 1.00
$$

For $\alpha=0.894, \mathrm{~W}(\alpha)=157.54$ and $\mu_{\mathrm{G}}=\mathrm{m} / \mathrm{W}(\alpha)=0.894$

Once the optimum $\alpha$ is known the sizing variables are obtained. The fuzzy optimum lead to a solution $1.2 \%$ lighter than the original deterministic solution.

If the tolerance of the stress limits is half and keeping the linear membership functions, the results obtained in the first phase are:

$$
\mathrm{M}=\mathrm{W}(1)=159.52 \quad \mathrm{~m}=\mathrm{W}(0)=149.16 \quad 0.935 \leq \alpha \leq 1.00
$$

The crisp solution obtained in the second phase:

$$
\alpha=0.94, \mathrm{~W}(\alpha)=158.74 \text { and } \mu_{\mathrm{G}}=\mathrm{m} / \mathrm{W}(\alpha)=0.94
$$

These results are only $0.5 \%$ lighter than the deterministic solution.

A 5\% imprecision of Young modulus was considered next. The solution reveals than when the stress (and not displacement) limits govern the optimum solution the influence of the Young modulus of the cables is negligible.

A $30 \%$ increase in the live load was also considered as traffic loading has significantly increased over the last decades. The results obtained in the level cuts phase are:

$$
\mathrm{M}=\mathrm{W}(1)=185.97 \quad \mathrm{~m}=\mathrm{W}(0)=159.52 \quad 0,818 \leq \alpha \leq 1
$$

The solution obtained in the second phase is $\alpha=0.873, \mathrm{~W}(\alpha)=182.62$ and $\mu_{\mathrm{G}}=$ $\mathrm{m} / \mathrm{W}(\alpha)=0.873$. The fuzzy optimum is $1.8 \%$ lighter than the original deterministic solution.

The same imprecision of the stress limits and loading are considered simultaneously next. If the fuzziness is represented by only one $\alpha$, meaning a perfect correlation between the increase in loading and acceptance of increased stress limits, the results obtained in the first phase are:

$$
\mathrm{M}=\mathrm{W}(1)=185.97 \quad \mathrm{~m}=\mathrm{W}(0)=140.88 \quad 0,758 \leq \alpha \leq 1
$$

For $\alpha=0.797, \mathrm{~W}(\alpha)=176.81$ and $\mu_{\mathrm{G}}=\mathrm{m} / \mathrm{W}(\alpha)=0.797$ and the fuzzy optimum gives a $4.9 \%$ lighter than the original deterministic solution.

If the overloading and acceptance of increased stress limits is considered by two independent $\alpha$ variables in the first phase:

$$
\mathrm{M}_{\mathrm{s}}=\mathrm{W}(1)=159.52 \quad \mathrm{~m}_{\mathrm{s}}=\mathrm{W}(0)=140.88 \quad 0,883 \leq \alpha_{\mathrm{s}} \leq 1
$$




$$
\mathrm{M}_{1}=\mathrm{W}(1)=167.33 \quad \mathrm{~m}_{1}=\mathrm{W}(0)=140.88 \quad 0,841 \leq \alpha_{1} \leq 1
$$

The solution obtained in the bound search phase is $\alpha_{\mathrm{s}}=0.894, \alpha_{1}=0.861$ and the fuzzy optimum is $\mathrm{W}\left(\alpha_{\mathrm{s}}, \alpha_{1}\right)=180.31$ which is $2 \%$ more costly than the previous solution but $3 \%$ lighter than the deterministic solution.

\section{Conclusions}

It is pointed out that the fuzzy optimization has the advantage of ordinary optimization with a more realistic model of fuzzy constraints taken into account, the structural design should be more reasonable and beneficial. The illustrative numerical examples given here show that the Two-Phase Method based on fuzzy set theory seems to be rational and effective approach for fuzzy optimization of structures with plastic or elastic material behaviour. As a result, the proposed approach provides favourable condition for selection of structural design schemes so as to have a high $\alpha$ level and to save materials.

\section{References}

[1] J.H.O. Negrão and L.M.C. Simões, "Optimization of Cable-stayed bridges with 3-dimensional modelling", Computers \& Structures, 64, pp.741-758, 1997.

[2] L.M.C. Simões and J.H.O. Negrão, "Optimization of Cable-stayed bridges subjected to earthquakes with non-linear behaviour", Engineering Optimization, 31, pp.457-478, 1999.

[3] J.H.O. Negrão and L.M.C. Simões, "Shape and sizing optimisation of boxgirder decks of cable-stayed bridges", Proc. OPTI 99 (Sixth Int. Conf. on Computer Aided Optimum Design of Structures), edts. S.Hernandez, A.J.Kassab \& C.A. Brebbia, WITpress, pp.323-332, 1999.

[4] J.H.O. Negrão and L.M.C. Simões, "Cable Stretching Force Optimization in Cable-stayed Bridges", Proc. WCSMO-2 (Second World Congress on Structural and Multidisciplinary Optimization), edts. W. Gutkowski and Z. Mroz, Zakopane May 26-30 Poland, Institute of Technological Recsearch, Warsaw,Poland, pp.983-988, 1997.

[5] E. Barbieri, C. Cinquini and M. Lombardi, "Shape/size Optimization of Truss Structures using Non-probabilistic Description of Uncertainty", Computer Aided Optimum Design of Structures V, C.A. Brebbia, and S. Hernandez, Ed., Springer-Verlag, 285-295, 1997

[6] G.-Y.Wang and W.-Q.Wang, "Fuzzy Optimum Design of Structures", Eng. Opt., 8, 291-300, 1985.

[7] J.L. Verdegay, "Fuzzy mathematical programming", Fuzzy Information and Decision Processes (M. M. Gupta and E. Sanchez ed.), pp. 231-237. NorthHolland, New York., 1982. 